\title{
Positive Solutions for Two-Point Boundary Value Problems for Fourth-Order Differential Equations with Fully Nonlinear Terms
}

\author{
Yixin Zhang $\mathbb{B D}^{1}$ and Yujun Cui $\mathbb{D}^{2}$ \\ ${ }^{1}$ School of Mathematics and Systems Science, Shandong University of Science and Technology, Qingdao, Shandong 266590, China \\ ${ }^{2}$ State Key Laboratory of Mining Disaster Prevention and Control Co-founded by Shandong Province \\ and the Ministry of Science and Technology, Shandong University of Science and Technology, Qingdao 266590, China
}

Correspondence should be addressed to Yujun Cui; cyj720201@163.com

Received 22 September 2020; Accepted 19 October 2020; Published 6 November 2020

Academic Editor: Chuanjun Chen

Copyright (c) 2020 Yixin Zhang and Yujun Cui. This is an open access article distributed under the Creative Commons Attribution License, which permits unrestricted use, distribution, and reproduction in any medium, provided the original work is properly cited.

In this paper, we consider the existence of positive solutions for the fully fourth-order boundary value problem $\left\{\begin{array}{l}u^{(4)}(t)=f\left(t, u(t), u^{\prime}(t), u^{\prime \prime}(t), u^{\prime \prime \prime}(t)\right), 0 \leq t \leq 1, \quad \text { where } \quad f:[0,1] \times[0,+\infty] \times(-\infty,+\infty) \times(-\infty, 0) \times(-\infty,+\infty) \longrightarrow \\ u(0)=u(1)=u^{\prime \prime}(0)=u^{\prime \prime}(1)=0\end{array}\right.$ $[0,+\infty]$ is continuous. This equation can simulate the deformation of an elastic beam simply supported at both ends in a balanced state. By using the fixed-point index theory and the cone theory, we discuss the existence of positive solutions of the fully fourthorder boundary value problem. We transform the fourth-order differential equation into a second-order differential equation by order reduction method. And then, we examine the spectral radius of linear operators and the equivalent norm on continuous space. After that, we obtain the existence of positive solutions of such BVP.

\section{Introduction}

In this paper, we study the existence of positive solutions for the fully fourth-order boundary value problem:

$$
\left\{\begin{array}{l}
u^{(4)}(t)=f\left(t, u(t), u^{\prime}(t), u^{\prime \prime}(t), u^{\prime \prime \prime}(t)\right), \quad 0 \leq t \leq 1, \\
u(0)=u(1)=u^{\prime \prime}(0)=u^{\prime \prime}(1)=0
\end{array}\right.
$$

where $f:[0,1] \times[0,+\infty] \times(-\infty,+\infty) \times(-\infty, 0) \times(-\infty$, $+\infty) \longrightarrow[0,+\infty]$ is continuous. This boundary value problem can simulate the deformation of an elastic beam, whose one end is fixed and the other end is free in a balanced state. In mechanics, BVP (1) is called a cantilever beam equation. In this equation, each derivative of $u(t)$ has its physical meaning: $u^{\prime}(t)$ is the slope, $u^{\prime \prime}(t)$ is the bending moment stiffness, $u^{\prime \prime \prime}(t)$ is the shear force stiffness, and $u^{(4)}(t)$ is the load density stiffness. The nonlinear fourthorder differential equation boundary value problem can simulate the deformation of an elastic beam under external force, and different boundary value conditions can show its force under different conditions. Because of its importance in mechanics, many scholars have done a lot of research on the existence of solutions for fourth-order ordinary differential equations using various nonlinear methods [1-11].

As the nonlinear term does not contain the derivative term of the unknown function, equation (1) becomes

$$
\left\{\begin{array}{l}
u^{(4)}(t)=f(t, u(t)), \quad 0 \leq t \leq 1 \\
u(0)=u(1)=u^{\prime \prime}(0)=u^{\prime \prime}(1)=0
\end{array}\right.
$$

If $f(t, u)$ is superlinear or sublinear growth on $u$, the authors in [1] used the fixed-point theorem on the cone to obtain the existence of the positive solution of equation (2). In [3], the author used the fixed-point theorem and topological degree theory to study the existence of one or two positive solutions for the fourth-order differential equation boundary value problem: 


$$
\left\{\begin{array}{l}
u^{(4)}(t)-\lambda f(t, u(t))=0, \quad 0 \leq t \leq 1, \\
u(0)=u(1)=u^{\prime \prime}(0)=u^{\prime \prime}(1)=0
\end{array}\right.
$$

Under the fixed-point index method on the cone, the authors of [11] discussed the existence of positive solutions for fourth-order boundary value problems with two parameters. Among them, the assumption condition of the nonlinear term $f$ is related to the first eigenvalue of the corresponding linear operator. It is noteworthy that the nonlinear term in the abovementioned boundary value problems does not include the higher-order derivative $u^{\prime \prime \prime}$. When the nonlinear term contains the higher-order derivative of the unknown function, the authors of $[8,9]$ used the upper and lower solution method to study the existence of solutions for fully fourth-order nonlinear boundary value problems with nonlinear boundary conditions. In [10], the author discussed a fourth-order boundary value problem with fully form:

$$
\left\{\begin{array}{l}
u^{(4)}(t)=f\left(t, u(t), u^{\prime}(t), u^{\prime \prime}(t), u^{\prime \prime \prime}(t)\right), \quad 0 \leq t \leq 1, \\
u(0)=u^{\prime}(0)=u^{\prime \prime}(1)=u^{\prime \prime \prime}(1)=0
\end{array}\right.
$$

When the nonlinear term satisfies superlinear growth and sublinear growth, the author used the fixed-point index method, combined with the positivity of linear operators and spectral radius, to get the positive solutions for the boundary value problem. But the linear operator in [10] does not involve the first and second derivatives of unknown functions.

In this paper, by using cone theory and the fixed-point index, combined with the spectral radius of linear integral operators, and the application of equivalent norms, we discuss the existence of positive solutions for boundary value problems (1).

\section{Preliminaries}

In this section, we give some assumptions that are important to our main results:

(i) $\left(H_{1}\right): f:[0,1] \times[0,+\infty] \times(-\infty,+\infty) \times(-\infty, 0) \times$ $(-\infty,+\infty) \longrightarrow[0,+\infty]$ is continuous

(ii) $\left(H_{2}\right)$ : there exist nonnegative constants $a_{1}, c_{1} \geq 0$ and $r>0$ such that $a_{1} x_{1}+c_{1} x_{3} \leq f\left(t, x_{1}, x_{2}-x_{3}\right.$, $\left.x_{4}\right), x_{1}, x_{3} \in[0, r], x_{2} \in[-r, r]$, and $x_{4} \in(-\infty,+\infty)$.

(iii) $\left(H_{3}\right)$ : there exist nonnegative constants $a, b, c$, $d, M \geq 0$ and $0<q<1$ such that

$$
\begin{array}{r}
f\left(t, x_{1}, x_{2},-x_{3}, x_{4}\right) \leq a x_{1}+b\left|x_{2}\right|+c x_{3}+d\left|x_{4}\right|^{q}+M, x_{1}, \\
x_{3} \in[0,+\infty], x_{2}, x_{4} \in(-\infty,+\infty) .
\end{array}
$$

Let $C[0,1]$ denote the Banach space of continuous functions from $[0,1]$ into $\mathbb{R}$ with norm $\|u\|=\max _{0 \leq t \leq 1}|u(t)|$. Let
$P=\{u \in C[0,1] u(t)$ is concave on $[0,1], u(0)=u(1)=0\}$. Then, $P$ is a positive cone on $C[0,1]$.

The functions on cone $P$ have the following properties:

Lemma 1 (see [12]). Every function $u \in P$ on the cone $P$ is differentiable almost everywhere on $(0,1)$ and satisfies

$$
\begin{gathered}
u(t) \geq t(1-t)\|u\|, \quad t \in[0,1], \\
\left|u^{\prime}(t)\right| \leq \frac{u(t)}{t(1-t)}, \quad \text { a.e. on }[0,1] .
\end{gathered}
$$

Let $v(t)=-u^{\prime \prime}(t)$, then the differential equation BVP (1) can be transformed into the following two second-order differential equations:

$$
\begin{gathered}
\left\{\begin{array}{l}
u^{\prime \prime}(t)=-v(t), \\
u(0)=u(1)=0,
\end{array}\right. \\
\left\{\begin{array}{l}
-v^{\prime \prime}(t)=f\left(t, u(t), u^{\prime}(t), u^{\prime \prime}(t), u^{\prime \prime}(t)\right), \\
v(0)=v(1)=0 .
\end{array}\right. \\
\text { Let } G(t, s)=\left\{\begin{array}{ll}
s(1-t) & 0 \leq s \leq t \leq 1 \\
t(1-s) & 0 \leq t \leq s \leq 1
\end{array},\right. \text { which is the cor- }
\end{gathered}
$$

responding Green's function of BVP (7). Thus, BVP (7) can be transformed into an equivalent integral equation:

$$
u(t)=\int_{0}^{1} G(t, s) v(s) \mathrm{d} s .
$$

By using (9), BVP (8) can be reduced to

$$
\left\{\begin{array}{l}
-v^{\prime \prime}(t)=f\left(t,(A v)(t),(A v)^{\prime}(t),-v(t),-v^{\prime}(t)\right), \\
v(0)=v(1)=0,
\end{array}\right.
$$

where operator $A$ is defined as $(A v)(t)=\int_{0}^{1} G(t, s) v(s) \mathrm{d} s$. Thus, BVP (10) can be reduced to the equivalent integral equation:

$$
v(t)=\int_{0}^{1} G(t, s) f\left(s,(A v)(s),(A v)^{\prime}(s),-v(s),-v^{\prime}(s)\right) \mathrm{d} s .
$$

From the expression of Green's function $G(t, s)$, we know that $G(t, s)$ is continuous on $[0,1] \times[0,1]$, and we have

$$
t(1-t) s(1-s) \leq G(t, s) \leq s(1-s) \text { or } t(1-t), \quad 0 \leq t, s \leq 1 .
$$

From the standard proof, we can easily obtain the following statement.

Lemma 2. $A: L[0,1] \longrightarrow C[0,1]$ is a completely continuous operator.

For $\forall v \in P$, we define an operator $(F v)(t)=f(t$, $\left.(A v)(t),(A v)^{\prime}(t),-v(t),-v^{\prime}(t)\right)$. It follows from Lemma 1 and condition $\left(\mathrm{H}_{3}\right)$ that 


$$
\begin{aligned}
|(F v)(t)| & =\left|f\left(t,(A v)(t),(A v)^{\prime}(t),-v(t),-v^{\prime}(t)\right)\right| \\
& \leq a(A v)(t)+b\left|(A v)^{\prime}(t)\right|+c v(t)+d\left|v^{\prime}(t)\right|^{q}+M \\
& \leq a \int_{0}^{1} G(t, s) v(s) \mathrm{d} s+b \int_{0}^{1}\left|G_{t}(t, s)\right| v(s) \mathrm{d} s+c v(t)+d \frac{\|v\|^{q}}{t^{q}(1-t)^{q}}+M .
\end{aligned}
$$

It shows that $F$ is an operator which is defined on $P$ to $L(0,1)$. It is easy to conclude that $F$ is the continuous bounded operator mapping from $P$ to $L(0,1)$. Therefore, $T=A^{\circ} F: P \longrightarrow P$ is a completely continuous operator. So, BVP (1) is equivalent to the operator equation $u=T u$, where the operator $T=P \longrightarrow P$ is given by

$$
(T v)(t)=\int_{0}^{1} G(t, s) f\left(s,(A v)(s),(A v)^{\prime}(s),-v(s),-v^{\prime}(s)\right) \mathrm{d} s .
$$

Let $\tilde{a}=(a, b, c)$ with $a, b, c \geq 0$. We define an operator $T_{\widetilde{a}}$ on cone $P$.

$$
\begin{aligned}
\left(T_{\widetilde{a}} u\right)(t)= & a \int_{0}^{1} G(t, s) \int_{0}^{1} G(s, \tau) u(\tau) \mathrm{d} \tau \mathrm{d} s \\
& +b \int_{0}^{1} G(t, s) \int_{0}^{1}\left|G_{s}(s, \tau)\right| u(\tau) \mathrm{d} \tau \mathrm{d} s \\
& +c \int_{0}^{1} G(t, s) u(s) \mathrm{d} s, \quad u \in P .
\end{aligned}
$$

It is easy to see that $T_{\widetilde{a}}: P \longrightarrow P$ is a linear operator. Let $u_{0}(t)=t(1-t)$, by (15), we obtain

$$
\begin{aligned}
& \left(T_{\widetilde{a}} u_{0}\right)(t) \geq a t(1-t) \int_{0}^{1} s(1-s) \int_{0}^{1} G(s, \tau) \tau(1-\tau) \mathrm{d} \tau \mathrm{d} s+b t(1-t) \\
& \cdot \int_{0}^{1} s(1-s) \int_{0}^{1}\left|G_{s}(s, \tau)\right| \tau(1-\tau) \mathrm{d} \tau \mathrm{d} s+c t(1-t) \int_{0}^{1} s(1-s) s(1-s) \mathrm{d} s=t(1-t) \\
& \cdot\left[a \int_{0}^{1} s(1-s) \int_{0}^{1} G(s, \tau) \tau(1-\tau) \mathrm{d} \tau \mathrm{d} s+b \int_{0}^{1} s(1-s) \int_{0}^{1}\left|G_{s}(s, \tau)\right| \tau(1-\tau) \mathrm{d} \tau \mathrm{d} s+c \int_{0}^{1} s^{2}(1-s)^{2} \mathrm{~d} s\right] .
\end{aligned}
$$

Then, if scalars $a, b, c \geq 0$, not all equal to zero, there exists a constant $\alpha>0$ satisfies $T_{\widetilde{a}} u_{0} \geq \alpha u_{0}$. Thus, from Krein-Rutman theorem, we know $r\left(T_{\widetilde{a}}\right)>0$, and there is $v_{\widetilde{a}} \in P /\{\theta\}$ such that $\lambda_{\widetilde{a}} T_{\widetilde{a}} v_{\widetilde{a}}=v_{\widetilde{a}}$, where $\lambda_{\widetilde{a}}=\left(r\left(T_{\widetilde{a}}\right)\right)^{-1}$ is the first eigenvalue of the operator $T_{\widetilde{a}}$.

Now, we estimate the range of $\lambda_{\widetilde{a}}=\left(r\left(T_{\widetilde{a}}\right)\right)^{-1}$. For any $u \in P$, from (15), we get

$$
\begin{aligned}
\left|\left(T_{\widetilde{a}} u\right)(t)\right| \leq & a \int_{0}^{1} s(1-s) \int_{0}^{1} G(s, \tau) u(\tau) \mathrm{d} \tau \mathrm{d} s \\
& +b \int_{0}^{1} s(1-s) \int_{0}^{1}\left|G_{s}(s, \tau)\right| u(\tau) \mathrm{d} \tau \mathrm{d} s \\
& +c \int_{0}^{1} s(1-s) u(s) \mathrm{d} s, \quad a, b, c \geq 0, \widetilde{a}=(a, b, c)
\end{aligned}
$$

thereby

$$
\begin{aligned}
\left\|T_{\widetilde{a}} u\right\| \leq & a \int_{0}^{1} s(1-s) \int_{0}^{1} G(s, \tau) u(\tau) \mathrm{d} \tau \mathrm{d} s \\
& +b \int_{0}^{1} s(1-s) \int_{0}^{1}\left|G_{s}(s, \tau)\right| u(\tau) \mathrm{d} \tau \mathrm{d} s \\
& +c \int_{0}^{1} s(1-s) u(s) \mathrm{d} s .
\end{aligned}
$$

By the expression of Green's function $G(t, s)$ and (15), we infer that

$$
\begin{aligned}
\left(T_{\widetilde{a}} u\right)(t) \geq & t(1-t)\left[a \int_{0}^{1} s(1-s) \int_{0}^{1} G(s, \tau) u(\tau) \mathrm{d} \tau \mathrm{d} s\right. \\
& +b \int_{0}^{1} s(1-s) \int_{0}^{1}\left|G_{s}(s, \tau)\right| u(\tau) \mathrm{d} \tau \mathrm{d} s \\
& \left.+c \int_{0}^{1} s(1-s) u(s) \mathrm{d} s\right], \quad a, b, c \geq 0, \tilde{a}=(a, b, c) .
\end{aligned}
$$

According to the above two inequalities, we have

$$
\left(T_{\widetilde{a}} u\right)(t) \geq t(1-t)\left\|T_{\widetilde{a}} u\right\|
$$

Recalled that $u_{0}(t)=t(1-t)$. By $(20)$, we have

$$
T_{\widetilde{a}} u_{0} \geq\left\|T_{\widetilde{a}} u_{0}\right\| u_{0} .
$$

Note the positivity of operator $T_{\widetilde{a}}$, we have $T_{\widetilde{a}}^{2} u_{0} \geq\left\|T_{\widetilde{a}} u_{0}\right\|^{2} u_{0}$. Hence, for any $n \in N$, using the recursion method to the above inequality, we get $T_{\widetilde{a}}^{n} u_{0} \geq\left\|T_{\widetilde{a}} u_{0}\right\|^{n} u_{0}$. Therefore, we have

$$
\left\|T_{\widetilde{a}}^{n} u_{0}\right\| \geq\left\|T_{\widetilde{a}} u_{0}\right\|^{n}\left\|u_{0}\right\| .
$$

Thus, 


$$
\left\|T_{\widetilde{a}}\right\|^{n} \geq \frac{\left\|T_{\tilde{a}}^{n} u_{0}\right\|}{\left\|u_{0}\right\|} \geq\left\|T_{\widetilde{a}} u_{0}\right\|^{n}
$$

From this inequality and Gelfand formula on spectral radius, we obtain

$$
r\left(T_{\tilde{a}}\right)=\lim _{n \longrightarrow \infty} \sqrt[n]{\left\|T_{\tilde{a}}^{n}\right\|} \geq\left\|T_{\widetilde{a}} u_{0}\right\|>0 .
$$

From (24), we get

$$
\left\|T_{\widetilde{a}}\right\| \geq r\left(T_{\tilde{a}}\right) \geq\left\|T_{\widetilde{a}} u_{0}\right\| .
$$

By $\lambda_{\tilde{a}}=\left(r\left(T_{\widetilde{a}}\right)\right)^{-1}$, we can get

$$
\frac{1}{\left\|T_{\widetilde{a}} \mathcal{u}_{0}\right\|} \leq \lambda_{\widetilde{a}} \leq \frac{1}{\left\|T_{\bar{a}}\right\|}
$$

Now, we calculate $\left\|T_{\widetilde{a}}\right\|$ and $\left\|T_{\widetilde{a}} u_{0}\right\|$ as follows. We have known that

$$
\begin{aligned}
G(s, \tau) & = \begin{cases}s(1-\tau), & 0 \leq s \leq \tau \leq 1, \\
\tau(1-s), & 0 \leq \tau \leq s \leq 1,\end{cases} \\
G_{s}(s, \tau) & = \begin{cases}1-\tau, & 0 \leq s \leq \tau \leq 1, \\
-\tau, & 0 \leq \tau<s \leq 1 .\end{cases}
\end{aligned}
$$

We first calculate $\left\|T_{\widetilde{a}}\right\|$. From (15), we get

$$
\begin{aligned}
\left\|T_{\widetilde{a}}\right\| & =\max _{t \in[0,1]} a \int_{0}^{1} G(t, s) \int_{0}^{1} G(s, \tau) u(\tau) \mathrm{d} \tau \mathrm{d} s \\
& +b \int_{0}^{1} G(t, s) \int_{0}^{1}\left|G_{s}(s, \tau)\right| u(\tau) \mathrm{d} \tau \mathrm{d} s \\
& +c \int_{0}^{1} G(t, s) u(s) \mathrm{d} s .
\end{aligned}
$$

Through integration, we have

$\left\|T_{\widetilde{a}}\right\|=\max _{t \in[0,1]}\left[t(1-t)\left(\frac{1}{12} a+\frac{1}{3} b+\frac{1}{2} c\right)\right]=\frac{1}{48} a+\frac{1}{12} b+\frac{1}{8} c$.

Next, we calculate $\left\|T_{\widetilde{a}} u_{0}\right\|$. Because

$$
\begin{aligned}
\left\|T_{\widetilde{a}} u_{0}\right\|= & \max _{t \in[0,1]}\left[a \int_{0}^{1} G(t, s) \int_{0}^{1} G(s, \tau) u_{0}(\tau) \mathrm{d} \tau \mathrm{d} s\right. \\
& +b \int_{0}^{1} G(t, s) \int_{0}^{1}\left|G_{s}(s, \tau)\right| u_{0}(\tau) \mathrm{d} \tau \mathrm{d} s \\
& \left.+c \int_{0}^{1} G(t, s) u_{0}(s) \mathrm{d} s\right] .
\end{aligned}
$$

Through integration, we have

$$
\begin{aligned}
\left\|T_{\widetilde{a}} \mathcal{u}_{0}\right\| & =\max _{t \in[0,1]}\left[t(1-t)\left(\frac{1}{60} a+\frac{1}{15} b\right)+\left(\frac{1}{12} t-\frac{1}{6} t^{3}+\frac{1}{12} t^{4}\right) c\right] \\
& =\frac{1}{240} a+\frac{1}{60} b+\frac{5}{192} c .
\end{aligned}
$$

Combining this with (29), we can obtain

$$
\frac{48}{a+4 b+6 c} \leq \lambda_{\tilde{a}} \leq \frac{960}{4 a+16 b+25 c}, \quad a, b, c>0, \tilde{a}=(a, b, c) .
$$

Now, we consider the special case with $\tilde{b}=\left(a_{1}, 0, c_{1}\right)$. We make a definition that

$$
\begin{aligned}
\left(T_{\widetilde{b}} u\right)(t)= & a_{1} \int_{0}^{1} G(t, s) \int_{0}^{1} G(s, \tau) u(\tau) \mathrm{d} \tau \mathrm{d} s \\
& +c_{1} \int_{0}^{1} G(t, s) u(s) \mathrm{d} s, \quad \tilde{b}=\left(a_{1}, 0, c_{1}\right) .
\end{aligned}
$$

Because of $\lambda_{\vec{b}} T \sim v_{b}(t)=v_{b}(t)$, it is equivalent to $\lambda_{\vec{b}}$ and $v_{b}(t)$ satisfying the differential equation:

$$
\left\{\begin{array}{l}
u^{(4)}(t)=\lambda_{b}\left[a_{1} u(t)-c_{1} u^{\prime \prime}(t)\right], \quad t \in[0,1] \\
u(0)=u(1)=u^{\prime \prime}(0)=u^{\prime \prime}(1)=0
\end{array}\right.
$$

We notice that the function $v_{b}(t)$ is nonnegative. After solving the above differential equations, we may take $v_{b}(t)=\sin \pi t$. Hence, we conclude the value of $\lambda_{b}$ is $\lambda_{b}^{b}=\pi^{4} / a_{1}+\pi^{2} c_{1}$.

To prove our main results, we also need the two following lemmas.

Lemma 3 (see $[13,14]$ ). Let $E$ is a Banach space, $P$ is a cone in $E$, and $\Omega(P)$ is a bounded open set in P. Assume that $A: \overline{\Omega(P)} \longrightarrow P$ is a completely continuous operator. If there exists $x_{0} \in P /\{\theta\}$ such that

$$
x-A x \neq \mu x_{0}, \quad \forall x \in \partial \Omega(P), \mu \geq 0 .
$$

Then, $i(A, \Omega(P), P)=0$.

Lemma 4 (see $[13,14])$. Let $E$ is a Banach space, $P$ is a cone in $E, \Omega(P)$ is a bounded open set in $P$, and $\theta \in \Omega(P)$. Assume that $A: \overline{\Omega(P)} \longrightarrow P$ is a completely continuous operator. If

$$
A x \neq \mu x, \quad \forall x \in \partial \Omega(P), \mu \geq 1,
$$

then $i(A, \Omega(P), P)=1$.

\section{Main Results}

Theorem 1. Suppose that conditions $\left(H_{1}\right),\left(H_{2}\right)$, and $\left(H_{3}\right)$ are satisfied, and

$$
\begin{aligned}
& \lambda_{\tilde{a}}>1, \\
& \lambda_{\tilde{b}}<1 .
\end{aligned}
$$

$\lambda_{\tilde{a}}$ and $\lambda_{\tilde{b}}$ are the first eigenvalues of operator $T_{\tilde{a}}$ and operator $T_{\tilde{b}}$, respectively. Then, BVP (1) has at least one positive solution.

Proof. It follows from $\left(H_{1}\right)$ and $\left(H_{2}\right)$ that, for some $r>0$, the function $f$ satisfies 


$$
\begin{aligned}
f\left(t, x_{1}, x_{2},-x_{3}, x_{4}\right) & \geq a_{1} x_{1}+c_{1} x_{3}, \\
x_{1}, x_{3} & \in[0, r], \\
x_{2} & \in[-r, r], \\
x_{4} & \in(-\infty,+\infty) .
\end{aligned}
$$

It is known that $v_{b}$ is the positive eigenvector of $T_{\widetilde{b}}$ corresponding to $\lambda_{\tilde{b}}$, and we have $v_{b}=\lambda \sim T_{\tilde{b}} v_{b}$. According to (14), (38), and (39), for any $v \in \partial B_{r}^{b} \cap P$, we have

$$
\begin{aligned}
|(A v)(t)| & =\int_{0}^{1} G(t, s)|v(s)| \mathrm{d} s \leq r \int_{0}^{1} G(t, s) \mathrm{d} s<r, \\
\left|(A v)^{\prime}(t)\right| & =\int_{0}^{1}\left|G_{t}(t, s)\right||v(s)| \mathrm{d} s \leq r \int_{0}^{1}\left|G_{t}(t, s)\right| \mathrm{d} s<r .
\end{aligned}
$$

Thus, we get

$$
\begin{aligned}
(T v)(t)= & \int_{0}^{1} G(t, s) f\left(s,(A v)(s),(A v)^{\prime}(s),-v(s),-v^{\prime}(s)\right) \mathrm{d} s \\
\geq & \int_{0}^{1} G(t, s)\left(a_{1}(A v)(s)+c_{1} v(s)\right) \mathrm{d} s \\
= & a_{1} \int_{0}^{1} G(t, s) \int_{0}^{1} G(s, \tau) v(\tau) \mathrm{d} \tau \mathrm{d} s \\
& +c_{1} \int_{0}^{1} G(t, s) v(s) \mathrm{d} s=\left(T \widetilde{b}^{v}\right)(t), \quad t \in[0,1] .
\end{aligned}
$$

Let us suppose that $T$ has no fixed point on $\partial B_{r} \cap P$; otherwise, Theorem 1 is proved.

Now, we prove

$$
v-T v \neq \zeta v_{b}, \quad \forall v \in \partial B_{r} \cap P, \zeta \geq 0,
$$

where $B_{r}=\{x \in C[0,1] \mid\|x\| \leq r\}$. If otherwise, there exists $v_{0} \in \partial B_{r} \cap P$ and $\zeta_{0} \geq 0$ such that $v_{0}-T v_{0}=\zeta_{0} v_{b}$. Then, we have $\zeta_{0}>0$ and $v_{0}=T v_{0}+\zeta_{0} v_{b} \geq \zeta_{0} v_{b}$. Let $\zeta^{*}=\sup$ $\left\{\zeta \mid v_{0} \geq \zeta v_{b}\right\}$, we can know $\zeta^{*} \geq \zeta_{0}>0$ and $v_{0} \geq \zeta^{*} v_{b}$. By $T_{\widetilde{b}}(P) \subset P$, we get $\lambda_{b}^{-T} T_{b} v_{0} \geq \zeta^{*} \lambda_{b} T_{\widetilde{b}} v_{b}=\zeta^{*} v_{b}$. Based on (41), we have

$$
v_{0}=T v_{0}+\zeta_{0} v_{b} \geq T \widetilde{b} v_{0}+\zeta_{0} v_{b} \geq\left(\frac{\zeta^{*}}{\lambda_{\vec{b}}}+\zeta_{0}\right) v_{b}
$$

Then, by the definition of $\zeta^{*}$, we conclude that $\zeta^{*} \geq \zeta^{*} / \lambda_{b}+\zeta_{0}$. By (15), we notice that $\lambda_{b}<1$ and $\zeta_{0}>0$. So, the above inequality contradicts the definition of $\zeta^{*}$. Hence, (42) holds. By Lemma 3, we have

$$
i\left(T, B_{r} \cap P, P\right)=0 \text {. }
$$

From $\left(H_{3}\right)$, we have

$$
\begin{aligned}
(T v)(t) & \leq \int_{0}^{1} G(t, s)\left(a \int_{0}^{1} G(s, \tau) v(\tau) \mathrm{d} \tau+b \int_{0}^{1}\left|G_{s}(s, \tau)\right| v(\tau) \mathrm{d} \tau+c v(s)+d\left|v^{\prime}(s)\right|^{q}+M\right) \mathrm{d} s \\
& \leq a \int_{0}^{1} G(t, s) \int_{0}^{1} G(s, \tau) v(\tau) \mathrm{d} \tau \mathrm{d} s+b \int_{0}^{1} G(t, s) \int_{0}^{1}\left|G_{s}(s, \tau)\right| v(\tau) \mathrm{d} \tau \mathrm{d} s+c \int_{0}^{1} G(t, s) v(s) \mathrm{d} s+d \int_{0}^{1} G(t, s) \frac{|v(s)|^{q}}{s^{q}(1-s)^{q}} \mathrm{~d} s+M \\
& =\left(T_{\widetilde{a}} v\right)(t)+d \int_{0}^{1} G(t, s) \frac{|v(s)|^{q}}{s^{q}(1-s)^{q}} \mathrm{~d} s \leq\left(T_{\widetilde{a}} v\right)(t)+d \int_{0}^{1} G(t, s) \frac{|v(s)|^{q}}{s^{q}(1-s)^{q}} \mathrm{~d}+M \\
& \leq\left(T_{\widetilde{a}} v\right)(t)+d \int_{0}^{1} s(1-s) \frac{|v(s)|^{q}}{s^{q}(1-s)^{q}} \mathrm{~d} s+M \leq\left(T_{\widetilde{a}} v\right)(t)+d \int_{0}^{1}|v(s)|^{q} \mathrm{~d} s+M
\end{aligned}
$$

Note that $\left(r\left(T_{\widetilde{a}}\right)\right)^{-1}>1$. Take

$$
\varepsilon=\frac{1-r\left(T_{\widetilde{a}}\right)}{2} \text {. }
$$

According to Gelfand formula, $r\left(T_{\widetilde{a}}\right)=\lim _{n \longrightarrow \infty} \sqrt[n]{\left\|T_{\widetilde{a}}^{n}\right\|}$, and there is a natural number $N$, so that, for $n \geq N$, $\left\|T_{\tilde{a}}^{n}\right\| \leq\left[r\left(T_{\widetilde{a}}\right)+\varepsilon\right]^{n}$.

For any $v \in C[0,1]$, we define

$$
\|v\|^{*}=\sum_{i=1}^{N}\left[r\left(T_{\widetilde{a}}\right)+\varepsilon\right]^{N-i}\left\|T_{\tilde{a}}^{i-1} v\right\|,
$$

where $T_{1}^{0}=I$ is an identity operator. It is not difficult to verify that $\|\cdot\|^{*}$ is a norm in $C[0,1]$, and we have

$$
\left\|\int_{0}^{1} v^{q}(s) \mathrm{d} s\right\|^{*} \leq K\|v\|^{* q}, \quad v \in C[0,1],
$$

where $K$ is a constant. Take

$$
r_{1}>\max \left\{1, r, 4\|M\|^{*} \varepsilon^{-1},\left(4 d K \varepsilon^{-1}\right)^{1 / 1-q}\right. \text {. }
$$

Because of $\|v\|^{*} \geq\left[r\left(T_{\widetilde{a}}\right)+\varepsilon\right]^{N-1}\|v\|$, we can take $r_{2}>r_{1}$ so that when $\|v\| \geq r_{2}$, we have $\|v\|^{*}>r_{1}$. 
Next, we prove

$$
T v \neq \mu v, \quad \forall v \in \partial B_{r_{2}} \cap P, \mu \geq 1 .
$$

If there exists $v_{1} \in \partial B_{r_{2}} \cap P$ and $\mu_{0} \geq 1$, such that $T v_{1}=\mu_{0} v_{1}$, by Lemma 1 and (45), we can obtain

$$
\begin{aligned}
\left(T v_{1}\right)(t) & =\int_{0}^{1} G(t, s) f\left(s,\left(A v_{1}\right)(s),\left(A v_{1}\right)^{\prime}(s), v_{1}(s), v_{1}^{\prime}(s)\right) \mathrm{d} s \\
& \leq\left(T_{\widetilde{a}} v_{1}\right)(t)+d \int_{0}^{1} v_{1}^{q}(s) \mathrm{d} s+M .
\end{aligned}
$$

Hence, we have

$$
0 \leq \mu_{0} v_{1}(t)=\left(T v_{1}\right)(t) \leq\left(T_{\widetilde{a}} v_{1}\right)(t)+d \int_{0}^{1} v_{1}^{q}(s) \mathrm{d} s+M,
$$$$
\forall t \in[0,1] .
$$

Furthermore, $T_{\widetilde{a}}(P) \subset P$, and we have

$$
\begin{array}{r}
0 \leqq\left(T_{\widetilde{a}}^{j}\left(T v_{1}\right)\right)(t) \leq\left(T_{\widetilde{a}}^{j}\left(T_{\widetilde{a}} v_{1}+d \int_{0}^{1} v_{1}^{q}(s) \mathrm{d} s+M\right)\right)(t), \\
\forall t \in[0,1], j=1,2, \ldots, N-1,
\end{array}
$$

and we also get

$$
\begin{array}{r}
\left\|T_{\widetilde{a}}^{j}\left(T v_{1}\right)\right\| \leq\left\|T_{\widetilde{a}}^{j}\left(T_{\widetilde{a}} v_{1}+d \int_{0}^{1} v_{1}^{q}(s) \mathrm{d} s+M\right)\right\|, \\
j=1,2, \ldots, N-1 .
\end{array}
$$

Therefore, from (47), we have

$$
\begin{aligned}
\left\|T v_{1}\right\|^{*} & =\sum_{i=1}^{N}\left[r\left(T_{\widetilde{a}}\right)+\varepsilon\right]^{N-i}\left\|T_{\widetilde{a}}^{i-1}\left(T v_{1}\right)\right\| \\
& \leq \sum_{i=1}^{N}\left[r\left(T_{\widetilde{a}}\right)+\varepsilon\right]^{N-i}\left\|T_{\widetilde{a}}^{i-1}\left(T_{\widetilde{a}} v_{1}+d \int_{0}^{1} v_{1}^{q}(s) \mathrm{d} s+M\right)\right\| \\
& =\left\|T_{\widetilde{a}} v_{1}+d \int_{0}^{1} v_{1}^{q}(s) \mathrm{d} s+M\right\|^{*} .
\end{aligned}
$$

When $\left\|v_{1}\right\|=r_{2}$, we have $\left\|v_{1}\right\|^{*}>r_{1}$. Hence, from (48) and (55), it follows that

$$
\begin{aligned}
\mu_{0}\left\|v_{1}\right\|^{*} & =\left\|T v_{1}\right\|^{*} \leq\left\|T_{\widetilde{a}} v_{1}+d \int_{0}^{1} v_{1}^{q}(s) d s+M\right\|^{*} \leq\left\|T_{\widetilde{a}} v_{1}\right\|^{*}+\left\|d \int_{0}^{1} v_{1}^{q}(s) \mathrm{d} s\right\|^{*} \\
& \leq\left\|T_{\widetilde{a}} v_{1}\right\|^{*}+\mathrm{d} K\left\|v_{1}\right\|^{* q}+\frac{\varepsilon}{4}\left\|v_{1}\right\|^{*}=\sum_{i=1}^{N-1}\left[r\left(T_{\widetilde{a}}\right)+\varepsilon\right]^{N-i}\left\|T_{\widetilde{a}}^{i} v_{1}\right\|+\mathrm{d} K\left\|v_{1}\right\|^{* q}+\frac{\varepsilon}{4}\left\|v_{1}\right\|^{*} \\
& \leq\left[r\left(T_{\widetilde{a}}\right)+\varepsilon\right] \sum_{i=1}^{N-1}\left[r\left(T_{\widetilde{a}}\right)+\varepsilon\right]^{N-i-1}\left\|T_{\widetilde{a}}^{i} v_{1}\right\|+\left\|T_{\widetilde{a}}^{N} v_{1}\right\|+\frac{\varepsilon}{4}\left\|v_{1}\right\|^{* q} r_{1}^{1-q}+\frac{\varepsilon}{4}\left\|v_{1}\right\|^{*} \\
& \leq\left[r\left(T_{\widetilde{a}}\right)+\varepsilon\right] \sum_{i=1}^{N-1}\left[r\left(T_{\widetilde{a}}\right)+\varepsilon\right]^{N-i-1}\left\|T_{\widetilde{a}}^{i} v_{1}\right\|\left[r\left(T_{\widetilde{a}}\right)+\varepsilon\right]^{N}\left\|v_{1}\right\|+\frac{\varepsilon^{4}}{4}\left\|v_{1}\right\|^{*}+\frac{\varepsilon}{4}\left\|v_{1}\right\|^{*} \\
& \leq\left[r\left(T_{\widetilde{a}}\right)+\varepsilon\right] \sum_{i=1}^{N-1}\left[r\left(T_{\widetilde{a}}\right)+\varepsilon\right]^{N-i}\left\|T_{\widetilde{a}}^{i-1} v_{1}\right\|+\frac{\varepsilon}{2}\left\|v_{1}\right\|^{*} \\
& =\left[r\left(T_{\widetilde{a}}\right)+\varepsilon\right]\left\|v_{1}\right\|^{*}+\frac{\varepsilon_{2}}{2}\left\|v_{1}\right\|^{*} \leq\left[r\left(T_{\widetilde{a}}\right)+\frac{3 \varepsilon}{2}\right]\left\|v_{1}\right\|^{*} .
\end{aligned}
$$

By $\mu_{0} \geq 1$ and (56), we can get $1 \leq r\left(T_{\widetilde{a}}\right)+3 \varepsilon / 2$. But it is contradictory to the definition of $\varepsilon=1-r\left(T_{\widetilde{a}}\right) / 2$. Hence, (50) holds. According to Lemma 4, we obtain that

$$
i\left(T, B_{r_{2}} \cap P, P\right)=1 \text {. }
$$

Now, from (44) and (57), it follows that

$$
i\left(T, \frac{\left(B_{r_{2}} \cap P\right)}{\left(\overline{B_{r}} \cap P\right)}, P\right)=i\left(T, B_{r_{2}} \cap P, P\right)-i\left(T, B_{r} \cap P, P\right)=1 .
$$

Hence, $T$ has at least one fixed point in $\left(B_{r_{2}} \cap P\right) /$ $\left(\bar{B}_{r} \cap P\right)$. That is to say, BVP (1) has at least one positive solution. The proof of Theorem 1 is completed. 


\section{Data Availability}

The data used to support the findings of this study are included within the article.

\section{Conflicts of Interest}

The authors declare that they have no conflicts of interest.

\section{Acknowledgments}

The research was supported by the National Natural Science Foundation of China (11571207), Shandong Natural Science Foundation (ZR2018MA011), and the Tai'shan Scholar Engineering Construction Fund of Shandong Province of China.

\section{References}

[1] R. Ma and H. Wang, "On the existence of positive solutions of fourth-order ordinary differential equations," Applicable Analysis, vol. 59, no. 1-4, pp. 225-231, 1995.

[2] R. Dalmasso, "Uniqueness of positive solutions for some nonlinear fourth-order equations," Journal of Mathematical Analysis and Applications, vol. 201, no. 1, pp. 152-168, 1996.

[3] Z. Bai and H. Wang, "On positive solutions of some nonlinear fourth-order beam equations," Journal of Mathematical Analysis and Applications, vol. 270, no. 2, pp. 357-368, 2002.

[4] C. P. Gupta, "Existence and uniqueness results for the bending of an elastic beam equation at resonance," Journal of Mathematical Analysis and Applications, vol. 135, no. 1, pp. 208225, 1998.

[5] R. P. Agarwal, "On fourth order boundary value problems arising in beam analysis," Differential and Integral Equations, vol. 2, no. 1, pp. 91-110, 1989.

[6] Y. Liu and D. O'Regan, "Multiplicity results for a class of fourth order semipositonem-point boundary value problems," Applicable Analysis, vol. 91, no. 5, pp. 911-921, 2012.

[7] Y. Ma, Ch. Yin, and G. Zhang, "Positive solutions of fourthorder problems with dependence on all derivatives in nonlinearity under Stieltjes integral boundary conditions," Boundary Value Problems, vol. 2019, p. 41, 2019.

[8] F. Minhós, T. Gyulov, and A. I. Santos, "Lower and upper solutions for a fully nonlinear beam equation," Nonlinear Analysis: Theory, Methods \& Applications, vol. 71, no. 1-2, pp. 281-292, 2009.

[9] A. Cabada, J. Fialho, and F. Minhós, "Extremal solutions to fourth order discontinuous functional boundary value problems," Mathematische Nachrichten, vol. 286, no. 17-18, pp. 1744-1751, 2013.

[10] Y. Li, "Existence of positive solutions for the cantilever beam equations with fully nonlinear terms," Nonlinear Analysis: Real World Applications, vol. 27, pp. 221-237, 2016.

[11] Y. Li, "Positive solutions of fourth-order boundary value problems with two parameters," Journal of Mathematical Analysis and Applications, vol. 281, no. 2, pp. 477-484, 2003.

[12] N. B. Huy and T. D. Thanh, "Global continua of positive solutions for some boundary value problems," Demonstratio Mathematica, vol. 35, pp. 303-309, 2002.

[13] D. Guo and V. Lakshmikantham, Nonlinear Problems in Abstract Cones, Academic Press, Inc, New York, NY, USA, 1988.

[14] D. Guo, Nonlinear Fuctional Analysis, Shandong Science and Technology Press, Jinan, China, 1987. 